\title{
RELATIONS AMONG SOME CLOSED GRAPH AND OPEN MAPPING THEOREMS
}

BY

\section{WILHELM (WROCEAW)}

1. Introduction. In the recent paper [5], Byczkowski and Pol proved some closed graph and open mapping theorems for Cech-complete topological spaces. Their results imply the corresponding ones of Banach [1] and [2], Klee [11], Weston [19], Brown [3], and Pettis [12]-[14]. They do not imply some sharper results known for completely metrizable topological vector spaces (Pták [16] and [17], A. Robertson and W. Robertson [18]), locally compact topological groups (Kelley [10]), completely metrizable topological groups (Kelley [10], Husain [9]), and Cech-complete topological groups (Brown [4]). All theorems under consideration use the notions of nearly continuity and nearly openness and appear to be superficially similar. However, their proofs involve various techniques and sometimes apply deep incidental results.

In this paper we present a unified approach to the subject. It is based on the notion of $\Delta$-closed graph, which is stronger than that of closed graph and weaker than continuity (cf. Section 2). We use a construction due to Weston [19] and Byczkowski and Pol [5] to prove a $\Delta$-closed graph theorem for Cech-complete topological spaces (Theorem in Section 3). Then we derive from it the most general of the mentioned results (cf. Section 4). Our closed graph (Corollary 4) and open mapping (Corollary 7) theorems for Cech-complete topological groups improve previous theorems of this kind.

2. Functions with $\Delta$-closed graph. Let $E$ be a topological space. We start with two propositions concerning a convergence-type property of nets in the product space $E \times E$. The diagonal is denoted by $\Delta_{E}$. A set has diameter less than an open cover $\mathscr{C}$ if it is in some member of $\mathscr{C}$.

Proposition 1. Let $\left\{\left(a_{\sigma}, b_{\sigma}\right)\right\}$ be a net in $E \times E$. The following conditions are equivalent:

(i) for any open set $W \supset \Delta_{k}$ the points $\left(a_{\sigma}, b_{\sigma}\right)$ are eventually in $W$ (i.e., there is $\sigma_{0}$ such that $\left(a_{\sigma}, b_{\sigma}\right) \in W$ for all $\left.\sigma \geqslant \sigma_{0}\right)$; 
(ii) for any open cover $\mathscr{C}$ of $E$ the sets $\left\{a_{\sigma}, b_{\sigma}\right\}$ have eventually diameter less than \&. $^{\circ}$

Proof. (i) $\Rightarrow$ (ii). Given $\mathscr{C}$, apply (i) to

$$
W=\bigcup_{U \in \mathscr{\ell}} U \times U
$$

(ii) $\Rightarrow$ (i). Given $W$, there exists a $\mathscr{C}$ such that

$$
\bigcup_{U \in \mathscr{Q}} U \times U \subset W
$$

apply (ii) to $\mathscr{C}$.

If the conditions of Proposition 1 hold, we will write $\left(a_{\sigma}, b_{\sigma}\right) \rightarrow \Delta_{K}$.

In case $E$ is a regular $\left(=T_{s}\right)$ space, $\left(a_{\sigma}, b_{\sigma}\right) \rightarrow \Delta_{E}$ and $\left(a_{\sigma}, b_{\sigma}\right) \rightarrow(a, b)$ imply $(a, b) \in \Delta_{K}$. It turns out that the validity of this implication is char: acteristic for Urysohn spaces. $E$ is said to be a Urysohn space if any two distinct points $a, b \in E$ can be separated with open sets $U_{1}, D_{2} \subset D$ whose closures are disjoint (cf. Engelking [6], Problem 1.7.7).

Proposition 2. The following are equivalent:

(i) $D$ is a Urysohn space;

(ii) for any point $(a, b) \in(E \times E) \backslash \Delta_{E}$ there exist disjoint open sets $W_{1}, W_{2} \subset E \times E$ such that $(a, b) \in W_{1}$ and $\Delta_{E} \subset W_{2}$;

(iii) $\left(a_{\sigma}, b_{\sigma}\right) \rightarrow \Delta_{E}$ and $\left(a_{\sigma}, b_{\sigma}\right) \rightarrow(a, b)$ imply $(a, b) \in \Delta_{Z^{*}}$.

Proof. (i) $\Rightarrow$ (ii). Put $W_{1}=U_{1} \times U_{2}$ and $W_{2}=(E \times E) \backslash \bar{W}_{1} ; \Delta_{E} \subset W_{2}$ because $\Delta_{H} \cap \bar{W}_{1}=\Delta_{H} \cap\left(\bar{U}_{1} \times \bar{U}_{2}\right)=0$.

(ii) $\Rightarrow$ (i). There are open sets $U_{i} \subset E$ such that $(a, b) \in U_{1} \times U_{2} \subset W_{1}$; since $\left(\bar{U}_{1} \times \bar{U}_{2}\right) \cap \Delta_{E} \subset \bar{W}_{1} \cap W_{2}=0, \bar{U}_{1} \cap \bar{U}_{2}=0$.

The equivalence of (ii) and (iii) can be proved analogously to the characterization of Hausdorff spaces as those in which each net has at most one limit (cf. Engelking [6], Proposition 1.6.7).

Let $D, F$ be topological spaces, and let $f$ be a function on $E$ to $F$.

Definition. The function $f$ has a $\Delta$-closed graph if $\left(a_{\theta}, b_{\sigma}\right) \rightarrow \Delta_{E}$ and $\left(f\left(a_{\sigma}\right), f\left(b_{\sigma}\right)\right) \rightarrow(c, d)$ imply $(c, d) \in \Delta_{F^{*}}$.

Proposition 3. If $f$ has a 4-closed graph, then $f$ has a olosed graph.

Proof. $a_{\sigma} \rightarrow a$ and $f\left(a_{\sigma}\right) \rightarrow 0$ imply $(c, f(a)) \in \Delta_{F^{*}}$.

Proposition 4. Suppose $F$ is a Urysohn space. If $f$ is continuous, then $f$ has a $\Delta$-closed graph.

Proof. Given an open cover $\mathscr{C}$ of $F, f^{-1}(\mathscr{C})$ is an open cover of $E$. Hence, in view of Proposition 1, $\left(a_{\sigma}, b_{\sigma}\right) \rightarrow \Delta_{k}$ implies $\left(f\left(a_{\sigma}\right), f\left(b_{\sigma}\right)\right) \rightarrow \Delta_{F}$, which yields the assertion (see Proposition 2, (i) $\Rightarrow$ (iii)).

There are two important classes of - not necessarily continuous functions which have $\Delta$-closed graph. They will be pointed out in the successive two propositions. 
A function $f$ is called inversely subcontinuous if any net $\left\{a_{\sigma}\right\} \subset D$ has a convergent subnet provided $\left\{f\left(a_{\sigma}\right)\right\}$ is convergent (cf. Fuller [8]). This is a generalization of a function with a compact domain and of a one-to-one function onto (= bijection) with a continuous inverse.

LEMMA. Suppose that

(1) $f$ is inversely subcontinuous and has a closed graph.

Then for any net $\left\{\left(a_{\sigma}, b_{\sigma}\right)\right\} \subset E \times E$, which has no cluster points out of $\Delta_{E},\left(f\left(a_{\sigma}\right), f\left(b_{\sigma}\right)\right) \rightarrow(c, d)$ implies $(c, d) \in \Delta_{F}$.

Proof. Applying twice the inverse subcontinuity of $f$ we get a subnet of $\left\{\left(a_{\sigma}, b_{\sigma}\right)\right\}$ which converges to a point $(a, b) \in E \times E$. By the assumption on cluster points, $(a, b) \in \Delta_{E}$. Since $f$ has a closed graph, $c=f(a)$ and $d=f(b)$. Hence $c=d$.

Proposition 5. Suppose $E$ is a Urysohn space. If $f$ satisfies (1), then $f$ has a 4 -closed graph.

Proof. If $\left(a_{\sigma}, b_{\sigma}\right) \rightarrow \Delta_{E}$, the same property has any of its subnets; by Proposition 2 , (i) $\Rightarrow$ (iii), the net has no cluster points out of $\Delta_{K E}$. The assertion follows from the Lemma.

Proposition 6. Suppose that $E, F$ are topological groups and $f$ is a homomorphism. Then $f$ has a closed graph (if and) only if it has a 4 -closed graph.

Proof. $\left(a_{\sigma}, b_{\sigma}\right) \rightarrow \Delta_{E}$ yields $a_{\sigma} b_{\sigma}^{-1} \rightarrow 1_{E}$, and $\left(f\left(a_{\sigma}\right), f\left(b_{\sigma}\right)\right) \rightarrow(c, d)$ yields $f\left(a_{\sigma} b_{\sigma}^{-1}\right) \rightarrow c d^{-1}$; hence $c d^{-1}=f\left(1_{E}\right)=1_{F}$ (provided $f$ has a closed graph).

Now we will examine condition (1). Recall that a function is said to be closed if the image of any closed set is closed. A Tychonoff space $F$ is Cech-complete if it is a dense $G_{b}$ in a compact Hausdorff $\left(=T_{2}\right)$ space; locally compact spaces and completely metrizable spaces are Cech-complete (cf. Engelking [6], Section 3.9, and Theorem 4.3.26). A Hausdorff space $F$ is a $k$-space if it is an image of a locally compact space under a quotient mapping; this is so if $F$ is first-countable or Cech-complete (cf. Engelking [6], Section 3.3, and Theorem 3.9.5).

Proposition 7. Consider the following conditions $\left(E \in T_{2}\right)$ :

(2) $f$ is closed and the counter-image of any point is compact.

(3) $f$ has a closed graph and the counter-image of any compact set is compact.

Then $(1) \Leftrightarrow(2) \Rightarrow(3)$. If $F$ is a k-space, then also (3) $\Rightarrow(2)$.

Proof. (1) $\Rightarrow(2)$ and (1) $\Rightarrow(3)$. Let $K \subset F$ be compact and $\left\{a_{\sigma}\right\}$ a net in $f^{-1}(K)$. There is a subnet $\left\{a_{\sigma^{\prime}}\right\}$ such that $f\left(a_{\sigma^{\prime}}\right) \rightarrow c \in K$. Since $f$ is inversely 'subcontinuous, there is a subnet $\left\{a_{\sigma^{\prime \prime}}\right\}$ of $\left\{a_{\sigma^{\prime}}\right\}$ with $a_{\sigma^{\prime \prime}} \rightarrow a \in E$. The graph of $f$ is closed, so that $a \in f^{-1}(c) \subset f^{-1}(K)$. This shows that $f^{-1}(K)$ is compact. Similarly it can be proved that $f$ is closed. 
(2) $\Rightarrow(1)$. Let $f\left(a_{\sigma}\right) \rightarrow c \in F$. Put $A_{\sigma}=\left\{a_{\sigma^{\prime}}: \sigma^{\prime} \geqslant \sigma\right\}$ and $B_{\sigma}=\bar{A}_{\sigma} \cap f^{-1}(c)$ for all indices $\sigma$. By $(2), f\left(\bar{A}_{\sigma}\right) \supset \overline{f\left(A_{\sigma}\right)} \ni c$ and $f^{-1}(c)$ is compact. It follows that $\left\{B_{\sigma}\right\}$ is a family of compact sets having the finite intersection property; there is a point $b$ in its intersection $-b$ is, by definition, a cluster point of the net $\left\{a_{\theta}\right\}$ and $b \in f^{-1}(c)$. This yields both parts of (1).

Finally, let $F$ be a $k$-space and assume condition (3). Let $A \subset E$ be closed and $K \subset F$ compact. Given $c \in \overline{f(A) \cap K}$, choose a net $\left\{a_{\sigma}\right\} \subset$ $\subset A \cap f^{-1}(K)$ such that $f\left(a_{\sigma}\right) \rightarrow c$. Since $A \cap f^{-1}(K)$ is compact, there is a subnet of $\left\{a_{\sigma}\right\}$ which tends to a point $b$ in the set. The graph of $f$ is closed, so that $c=f(b) \in f(A) \cap K$. This proves that the intersection of $f(A)$ with any compact set $K$ is closed. By Theorem 3.3.18 from Engelking [6], $f(A)$ is closed.

3. Main result. A set is called nearly open if it is in the interior of its closure. A function is called nearly continuous (nearly open) if the counter-image (image) of any open set is nearly open (Pták [16] and [17]).

THEOREM. Let $E$ be a topological space, and let $F$ be a Cech-complete topological space. A mapping $f$ of $E$ to $F$ is continuous if (and only if) $f$ is nearly continuous and has a 4 -closed graph.

Proof. Let the letter $G$ stand for open sets in $F$. Since $F$ is a regular space and $f^{-1}(G) \subset \operatorname{Int} \overline{f^{-1}(G)} \subset \overline{f^{-1}(G)}$, it is sufficient to prove that $\overline{f^{-1}(G)} \subset f^{-1}(\bar{G})$. Put $\nabla_{0}=G$ and $W_{0}=F \backslash \bar{G}$ and assume, to get a contradiction, that $\overline{f^{-1}\left(V_{0}\right)} \cap f^{-1}\left(W_{0}\right) \neq 0$.

Let $\left\{\mathscr{B}_{n}\right\}$ be a sequence of open covers of $F$ such that any net $\left\{c_{\sigma}: \sigma \in \Sigma\right\} \subset F$ has a convergent subnet provided for each $n$ the family $\left\{\left\{c_{\sigma^{\prime}}: \sigma^{\prime} \geqslant \sigma\right\}: \sigma \in \Sigma\right\}$ contains sets of diameter less than $\mathscr{C}_{n}$. (Such a sequence of open covers of $F$ exists by a variant of Theorem 3.9.2 from Engelking [6] due to Frolik [7].) Following the proof of Theorem of Byczkowski and Pol [5] we can construct sequences $\left\{V_{n}\right\}$ and $\left\{W_{n}\right\}$ of open subsets of $F$ such that

(a) $\bar{V}_{n+1} \subset V_{n}$ and $\bar{W}_{n+1} \subset W_{n}, n=0,1,2, \ldots$;

( $\beta$ ) $\nabla_{n}$ and $W_{n}$ have diameter less than $\mathscr{C}_{n}, n \in N$;

$(\gamma) \overline{f^{-1}\left(\nabla_{n}\right)} \cap f^{-1}\left(W_{n}\right) \neq 0, n \in N$.

By $(\gamma)$, there exist nets $\left\{a_{n}^{\tau}: \tau \in T_{n}\right\} \subset f^{-1}\left(\nabla_{n}\right)$ and elements $b_{n} \in f^{-1}\left(W_{n}\right)$ such that $a_{n}^{\tau} \rightarrow b_{n}$. Consider the product index set

$$
\Sigma=N \times \prod_{i \in N} T_{i}
$$

$\left(\sigma \leqslant \sigma^{\prime}\right.$ iff $n \leqslant n^{\prime}$ and $\nu(i) \leqslant \nu^{\prime}(i)$ for all $i \in N$, where $\sigma=(n, v)$ and $\left.\sigma^{\prime}=\left(n^{\prime}, v^{\prime}\right)\right)$ and the corresponding product net

$$
\left\{\left(a_{\sigma}, b_{\sigma}\right): \sigma \in \Sigma\right\} \subset E \times E
$$

$\left(a_{\sigma}=a_{n}^{p(n)}\right.$ and $b_{\sigma}=b_{n}$ for $\left.\sigma=(n, v) \in \Sigma\right)$. 

index

Notice that if $U_{n} \subset E$ are open sets with $b_{n} \in U_{n}$, then there is an

$$
v_{0} \in \prod_{i \in N} T_{i}
$$

such that $a_{n}^{\gamma(n)} \in{J_{n}}_{n}$ for all $n \in N$ and $\nu \geqslant \nu_{0}$. The fact has two consequences. Firstly, $\left(a_{\sigma}, b_{\sigma}\right) \rightarrow \Delta_{E}$. Secondly, the net $\left\{\left(a_{\sigma}, b_{\sigma}\right)\right\}$ has no cluster points out of $\Delta_{E}$ provided $E$ is a Hausdorff space (this will be used in Corollary 1). Since $f\left(a_{n}^{\nu(n)}\right) \in \nabla_{n},(\alpha)$ and $(\beta)$ imply that the net $\left\{f\left(a_{\sigma}\right)\right\}$ has a subnet which converges to an element of $\nabla_{0}$. We have even more: any subnet of $\left\{f\left(a_{\sigma}\right)\right\}$ has a subnet which converges to an element of $\nabla_{0}$. The same holds for $\left\{f\left(b_{\sigma}\right)\right\}$ and $W_{0}$. Hence there exists a subnet $\left\{\left(a_{\sigma^{\prime}}, b_{\sigma^{\prime}}\right)\right\}$ of $\left\{\left(a_{o}, b_{\sigma}\right)\right\}$ such that $\left(f\left(a_{\sigma^{\prime}}\right), f\left(b_{\sigma^{\prime}}\right)\right) \rightarrow(c, d) \in \nabla_{0} \times W_{0^{\prime}}$. Clearly, $\left(a_{\sigma^{\prime}}, b_{o^{\prime}}\right) \rightarrow \Delta_{E^{*}}$. By the assumption that $f$ has a $\Delta$-closed graph, $(c, d) \in \Delta_{F}$. This gives a contradiction: $c=d \in \nabla_{0} \cap W_{0}=G \cap(F \backslash \bar{G})=0$.

4. Consequences. A mapping $f$ of $E$ to $F$ is called perfect if $E$ is a Hausdorff space and $f$ is continuous and satisfies condition (2) (cf. Engelking [6], Section 3.7).

Corollary 1 (cf. Byczkowski and Pol [5], Theorem and Corollary). Let $\boldsymbol{E}$ be a Hausdorff topological space, and let $\boldsymbol{F}$ be a Cech-complete topological space. A mapping $f$ of $E$ to $F$ is perfect if (and only if) $f$ is nearly continuous and satisfies condition (2) (equivalently, (1) or (3)).

Proof. If $E$ is a Urysohn space, the assertion follows immediately from the Theorem and Propositions 5 and 7. If $E$ is a Hausdorff space, we must apply the Lemma - instead of the assumption that $f$ has a $\Delta$-closed graph - in the final part of the proof of the Theorem.

Corollary 2 ([5]). Let $E$ and $F$ be as in Corollary 1 . An open bijection $f$ of $E$ onto $F$ is continuous if (and only if) $f$ is nearly continuous.

Corollary 3 ([5]). Let $\boldsymbol{E}$ and $F$ be Cech-complete topological spaces. $A$ mapping $f$ of $E$ to $F$ is continuous if (and only if) $f$ is nearly continuous and has a closed graph.

Proof. The graph $G(f) \subset E \times F$ is Cech-complete in its relative product topology (cf. Engelking [6], Theorems 3.9.8 and 3.9.6). The induced mapping $\varphi$ of $E$ onto $G(f)$, defined by $\varphi(x)=(x, f(x))$, fulfills the assumptions of Corollary 2. Hence $\varphi$ and $f$ are continuous.

The next corollary, which is a consequence of the Theorem and Proposition 6, extends the corresponding result of Kelley [10] (Problem 6.R: for $F$ completely metrizable left-complete, and for $F$ locally compact).

CoRoLlary 4. Let $E$ be a topological group, and let $F$ be a Cech-complete topological group. A homomorphism from $E$ to $F$ is continuous if (and only if) $f$ is nearly continuous and has a closed graph. 
Now we pass on to open mapping theorems. Corollary 2 yields

CoROLLARY 5 ([5]). Let $\boldsymbol{E}$ be a Hausdorff topological space, and let $F$ be a Cech-complete topological space. A continuous bijection $g$ of $F$ onto $\boldsymbol{B}$ is open if (and only if) $g$ is nearly open.

Corollary 3 yields

Corollary 6 ([5]). Let $E$ and $F$ be Cech-complete topological spaces. $A$ bijection $g$ of $F$ onto $B$ is open if (and only if) $g$ is nearly open and has a closed graph.

Corollaries 5 and 6 are not true for mappings which are not one-to-one; Byczkowski and Pol [5] gave an example of a continuous nearly open surjection $g: F \rightarrow E$ which is not open, where $F$ is separable and completely metrizable and $\boldsymbol{E}$ is the unit interval. We are only able to replace the assumptions that $g$ be an injection with the assumption that the quotient space $F / R(g)$ be Cech-complete and the quotient map $q: F \rightarrow \mid F / R(g)$ be open (where the equivalence relation $R(g)$ is the set of all pairs $(a, b) \in F \times F$ for which $g(a)=g(b))$. Our final result shows that, for homomorphisms between topological groups, the situation is much better.

CoROLLARY 7. Let $E$ be a topological group, and let $F$ be a Cech-complete topological group. A homomorphism $g$ from $F$ to $E$, with a closed kernel, is open if and only if $g$ is nearly open and has a closed graph.

Proof. The kernel Ker $g$ is an invariant subgroup of $F$, and the image $E_{0}=g\left(F^{\prime}\right)$ is a subgroup of $E$. Consider the quotient topological group $F_{0}=F /$ Ker $g$, the canonical 'quotient mapping $q: F \rightarrow F_{0}$, and the induced mapping $g_{0}: F_{0} \rightarrow E$. As well known, $q$ is a continuous open homomorphism and $g_{0}$ is a monomorphism.

Necessity. Assume $g$ is open. Then $E_{0}$ is open and closed, $g_{0}$ is open, $g_{0}^{-1}: E_{0} \rightarrow F_{0}$ continuous. Let $F \ni y_{\sigma} \rightarrow y \in F$ and $g\left(y_{\sigma}\right) \rightarrow x \in \mathbb{E}$. Then $x \in E_{0}, q\left(y_{\sigma}\right) \rightarrow q(y)$, and $q\left(y_{\sigma}\right)=g_{0}^{-1}\left(g\left(y_{\sigma}\right)\right) \rightarrow g_{0}^{-1}(x)$. Hence $x=g_{0}(q(y))$ $=g(y)$. Thus $g$ has a closed graph.

Sufficiency. Assume $g$ is nearly open and has a closed graph. (The last assumption yields that Ker $g$ is closed.) By Brown [4] (Theorem 2 and Corollary 3 to Theorem 1), $F_{0}$ is Cech-complete and complete in its two-sided uniformity. Since $g$ is nearly open and $q$ continuous, $g_{0}^{-1}$ is nearly continuous. Since $g$ has a closed graph and $q$ is open, one can prove that $g_{0}^{-1}$ has a closed graph. By Corollary $4, g_{0}^{-1}$ is continuous. Thus $g_{0}$ is open when considered as a map to $E_{0} ; g=g_{0} \circ q$ is open as a map to $E_{0}$. Now, it is sufficient to prove that $E_{0}$ is open in $E$. Since $E_{0}=g\left(F^{\prime}\right)$ is nearly open in $E$, it is sufficient to verify that $E_{0}$ is closed in $E$. Let $E_{0} \ni x_{\sigma} \rightarrow x \in E$. The net $\left\{g_{0}^{-1}\left(x_{\sigma}\right)\right\}$ is fundamental in $F_{0}$ with its two-sided uniformity, because $g_{0}^{-1}$ is a continuous isomorphism. Let $g_{0}^{-1}\left(x_{\sigma}\right) \rightarrow y_{0} \in F_{0}$. 
Since $g_{0}$ has a closed graph (as a subset of $\left.F_{0} \times E\right), x=g_{0}\left(y_{0}\right) \in E_{0}$. This completes the proof.

Corollary 7 contains the corresponding results of Kelley [10] (Problem 6.R : for $F$ completely metrizable left-complete, and for $F$ locally compact) and Brown [4] (Theorem 4: for $g$ continuous). It shows, in particular, that every Fréchet space ( = completely metrizable locally convex topological vector space) is a Pták space (=B-complete space in $[16]=$ fully complete space), and that every Cech-complete topological group is a $B(\mathscr{A})$-group in the sense of Husain [9], Chapter V.

\section{BIBLIOGRAPH $Y$}

[1] S. Banach Théorie des opérations linéaires, Warszawa 1932.

[2] - Uber metrische Gruppen, Studia Mathematica 3 (1931), p. 101-113.

[3] L. G. Brown, Note on the open mapping theorem, Pacific Journal of Mathematics 38 (1971), p. $25-28$.

[4] - Topologically complete groups, Proceedings of the American Mathematical Society 35 (1972), p. 593-600.

[5] T. B yozkowski and R. Pol, On the closed graph and open mapping theorems, Bulletin de l'Académie Polonaise des Sciences, Série des sciences mathématiques, astronomiques et physiques, 24 (1976), p. 723-726.

[6] R. Engelking, General topology, Warszawa 1977.

[7] Z. Frolik, Generalization of the $G_{\delta}$-property of complete metric space8, Czechoslovak Mathematical Journal 10 (1960), p. 255-257.

[8] R. V. Fuller, Relations among contimuous and various non-continuous functions, Pacific Journal of Mathematics 25 (1968), p. 495-509.

[9] T. Husain, Introduction to topological groups, Philadelphia 1966.

[10] J. L. Kelley, General topology, Princeton 1955.

[11] V. L. Klee, Invariant metrics in groups (solution of a problem of Banach), Proceedings of the American Mathematical Society 3 (1952), p. 484-487.

[12] B. J. Pettis, On continuity and openness of homomorphisms in topological groups, Annals of Mathematics 52 (1950), p. 293-308.

[13] - Oomments on open homomorphisms, Proceedings of the American Mathematical Society 8 (1957), p. 583-586.

[14] - Closed graph and open mapping theorems in certain topologically complete spaces, Bulletin of the London Mathematical Society 6 (1974), p. 37-41.

[15] - Addition to the note: Closed graph and open mapping theorems in certain topologically complete spaces, ibidem 7 (1975), p. $175-176$.

[16] V. Pták, On complete topological linear spaces, Czechoslovak Mathematioal Journal 78 (1953), p. $301-360$.

[17] - Completeness and the open mapping theorem, Bulletin de la Sociéte Mathématique de France 86 (1958), p. $41-74$.

[18] A. Robertson and W. Robertson, On the closed graph theorem, Proceedings of the Glasgow Mathematical Association 3 (1956), p. 9-12.

[19] J. D. Weston, On the comparison of topologies, Journal of the London Mathematical Society 32 (1957), p. $342-354$. 
[20] M. Wilhelm, On olosed graph theorems, Proceedings of the Fourth Prague Topological Symposium, 1976, Contributed Papers, p. 517-520.

[21] - On closed graph theorems in topological spaces and groups, Fundaments Mathematioae 104 (1978), p. 11-21.

INSTITUTH OF MATHEMATICS, WROCEAW TEOHNIOAL UNIVIERSITY

Regu par la Rédaction lo 3. 10. 1977 